\title{
Safe zones for shock-protection of fragile components during impact-induced clatter
}

\author{
Suresh Goyal ${ }^{\mathrm{a}}$ and James M. Papadopoulos ${ }^{\mathrm{b}}$ \\ ${ }^{a}$ Network Hardware Integration Research, Lucent Technologies, Bell Laboratories, 600 Mountain Avenue, Rm. \\ 1B-212, Murray Hill, NJ 07974 \\ Tel.: +1 908582 5959; Fax: +1 908582 6228; E-mail: goyal@lucent.com \\ ${ }^{\mathrm{b}}$ Paper Converting Machine Company, 2300 South Ashland Avenue, Green Bay, WI 54307 \\ Tel.: +1 920491 6763; E-mail: papadopoulos@alum.mit.edu
}

Received 29 December 1999

Revised 31 January 2001

\begin{abstract}
Clattering motion that occurs when flat objects strike the ground at an oblique angle is studied through a simple, tractable, model of a rigid bar with arbitrary, but symmetric, mass distribution and coefficient of restitution. The maximum velocity changes, or velocity shocks, that occur at various locations of the bar as it clatters to rest, are presented. It is shown that different parts of the bar can be subjected to sequences of velocity changes that are both higher, and lower, than those encountered in a single clatter-free impact. The implication that the drop-tolerance of an electronic product can be increased by configuring it to have 'safe zones' - where the velocity shocks are lower - for the placement of fragile components, is analysed. It is shown, through example, that a significant safe zone can be created in the center of the product by configuring it to have a low moment of inertia and by minimizing coefficient of restitution.
\end{abstract}

\section{Introduction}

Impact-tolerance, or the ability to safely withstand accidental drops and bangs against unyielding surfaces, is becoming increasingly important for portable electronic products like wireless web-surfing devices, laptop computers, cellular phones, personal digital assistants, etc. It enhances consumer confidence and reliance on these products, thereby creating greater market opportunities for manufactureres and service providers. An important factor that determines the outcome of an accidental drop of a portable product is the orientation of the object at impact.

Invariably, products drop so that one corner touches down first, followed by a 'clattering' as succesive corners strike one or more times, before the object either bounces clear, or comes to rest on the floor (for example, see [9]). However the classical treatment of single impact, for assessing impact-tolerance of products, does not address the repurcussions of clattering motion.
Where impact excites dynamic response of an internal degree of freedom, the nature of clattering (based on geometry, mass distribution, and restitution) is absolutely essential to engineering a product's durability.

Clattering behaviour was identified and modeled in previous work ([6,7]) for a bar with symmetric, yet arbitrary, mass distribution. This analysis showed that as a result of clattering, different parts of a product can be subjected to sequences of velocity changes that are both substantially higher, and lower, than those encountered in a single clatter-free impact. This suggests that the drop-tolerance of an electronic product can be increased by configuring it to have 'safe zones' - where the velocity shocks are lower - for the placement of fragile components.

In this paper, we continue our study of the smallangle clattering of a flat body. The main aim of this work is to show how the theoretical results presented in earlier work $([6,7])$ can be applied to actual problems confronting designers. The goal is to provide a simpli- 
fied recipe, so the user can avoid becoming enmeshed in deciphering and weighing the various parts of the relatively general analysis of $[6,7]$.

The layout of the paper is as follows. For completeness, the equations of motion for a clattering bar, derived in detail in [6], are summarized briefly, though heuristically. The concept of velocity shocks, and their relevance to shock analysis, is then explained. Maximal velocity changes at various locations of the bar, obtained using the above equation, are examined, both in global parameter space and with a specific example. Some product configurations to increase drop-tolerance are presented.

\section{Model and justification}

Small-angle clattering motion of an actual product will primarily involve rotations about axes parallel to the ground plane. Hence, we have focussed on the simplest model system that represents the above - a near-horizontal rod or bar which impacts the ground at its ends only, as shown in Fig. 1 - and is capable of displaying complex clattering motion and its repercussions for impact-tolerance. (It is usually recommended [9]) that for increased durability portable electronic products have a rigid outer housing, so our model of a rigid-bar is not inappropriate.)

There is ample reason to expect that rod-like behavior will commonly be observed for flat rectangular bodies of a variety of aspect ratios. For example, for the first few (large amplitude) clatters, a square plate will clatter on the initially-lowest corner and its diagonal opposite, until lowered enough for the other corners to take their turn. And a more rectangular plate will typically undergo several clatters involving the lowest corner and its 'fast' neighbor at the other end of a short side. Corroborative experimental examples are presented in [4,9].

The explicit extension of our study to the clattering of a plate, although a worthwhile endeavor, is beyond the scope of the current paper. It is not expected that the added (geometric and inertial) parameter dimensionality would add much in the way of insight, although it would certainly aggravate the chore of extracting design guidance.

\section{Equation of motion}

In this section we give a more concise and intuitive development of equations presented in ([6]).
Consider a near-horizontal rod approaching the ground at one of its ends, as shown in Fig. 1. The rod's mass distribution is symmetric but otherwise arbitrary. The impact of the ends is governed by a coefficient of restitution. The rod is assumed to be stiff enough that its fundamental period of free vibration is considerably shorter than any other timescale under consideration.

The bar is assumed to impact the ground at its ends only, hence each impact in the impact-sequence that arises is an 'eccentric impact', i.e. the impact-force exerts a moment about the center of mass (CM) of the bar and causes it to rotate. The net result of an eccentric impact is that various points on the bar alter their velocities by differing amounts, and one point does not alter its velocity at all - this is defined as the center of percussion (CP) with respect to the impact point ([2]). The CP is a key concept in calculating, and visualizing, the maximum velocity changes that occur during clattering of an object. The $\mathrm{CP}$ of a slender body, with respect to impacts at distance $L$ from the $\mathrm{CM}$, is at the distance $p=J / m L$ to the other side of the CM, where $m$ is the mass of the body, and $J$ is its moment of inertia about the CM.

The motion of the bar is described by the upwards velocities of its two endpoints, written as a vector $\mathbf{v}=$ $\left[v_{a}, v_{b}\right]$. Impacts in the clattering sequence are indexed with parameter $i$, with the additional convention that $X_{i}$ refer to the post-impact value of any quantity $X$, and $X_{i-1}$ represents its pre-impact value. For example, $v_{i}$ represents the velocity after the $i$ th impact, and $\Delta v_{i}=$ $v_{i}-v_{i-1}$ is the change in velocity due to the $i$ th impact.

As shown in [6], the equations of momentum conservation and energy restoration can be used to determine the endpoint velocities of the bar just after one end bounces, in terms of their values just before the bounce. For a left-end bounce (where $v_{a}<0$ ) this takes the form of a matrix multiplication by

$$
\mathbf{A} \equiv\left[\begin{array}{cc}
-e_{e} & 0 \\
q\left(1+e_{e}\right) & 1
\end{array}\right]
$$

to give,

$$
\begin{aligned}
\mathbf{v}_{1} & =\left[\begin{array}{l}
v_{a, 1} \\
v_{b, 1}
\end{array}\right]=\left[\begin{array}{cc}
-e_{e} & 0 \\
q\left(1+e_{e}\right) & 1
\end{array}\right]\left[\begin{array}{l}
v_{a, 0} \\
v_{b, 0}
\end{array}\right] \\
& =\mathbf{A v}_{0}
\end{aligned}
$$

Here, $0 \leqslant e_{e} \leqslant 1$ is the coefficient of restitution, or in other words the ratio of departure speed to approach speed of either end. (A constant $e_{e}$ is widely viewed as the simplest rigid-body impact model capable of reasonably representing real-world energy loss, despite its well known shortcomings [2, 


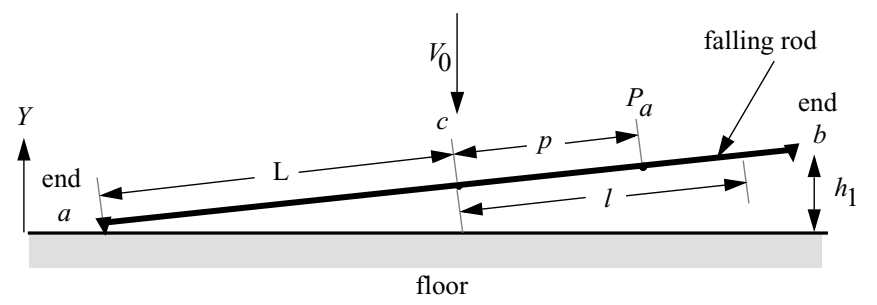

Fig. 1. Schematic of a bar falling vertically without rotation in an almost-horizontal configuration, and striking the ground with its left end, $a$, first. As a result of this collision, the bar is set into rotation, and experiences a rapid sequence of alternating-end impacts before it bounces free of the ground. We call this rapid sequence of impacts, which make up a single rebound, 'clattering'. The dynamics of this motion are governed primarily by the mass distribution of the bar and its coefficient of restitution. The point $P_{a}$ represents the center of precussion for impacts at $a$. For impacts at end $b$, the center of percussion, $P_{b}$, is at a distance $p$ to the other side of the CM.

15].) $q \equiv\left(L^{2}-\rho_{g}^{2}\right) /\left(L^{2}+\rho_{g}^{2}\right)$ is an inertial parameter which effectively relates radius of gyration $\rho_{g}$ to bar half-length $L$. (So given $\left.q, \rho_{g}=L \sqrt{(1-q) /(1+q)}\right)$.

The parameter $q$ is the negative ratio of the velocity jump at the non-impacting end, to the velocity jump at the impacting end ([6]). Depending as the contact point (i.e., length) is outboard, at, or inboard of the center of percussion for impacts at the other end, $q$ will be positive, zero, or negative. When $q=1$ all the mass is at the center of the bar. When $q=0$ all the mass is concentrated at the ends. And $q=0.5$ represents a radius of gyration of $L / \sqrt{3}$, for example a uniform mass distribution. While $q>1$ is impossible, $q<0$ is possible but unlikely - there must be considerable mass beyond the contacting endpoints.

After one end strikes, its velocity is positive and it will move up away from the ground. But if the other end now has negative velocity, it will soon strike in turn. For the very short times and large velocity jumps considered here, gravity is assumed not to act, so the endpoint velocities, found just after one end strikes, are used as the input to the next strike.

From A we can construct an iterated operator, which is applied repeatedly to the initial velocity conditions, until the non-impacting end retains non-negative velocity even after an impact at the other end. (In that case clattering ceases.)

The operator we need is PA, where

$$
\mathbf{P}=\mathbf{P}^{T}=\mathbf{P}^{-1}=\left[\begin{array}{ll}
0 & 1 \\
1 & 0
\end{array}\right]
$$

is the permutation matrix, which interchanges the $a$ and $b$ components of $\mathbf{v}$. The composite action represents an impact of the left end, followed by an interchange of the two components of velocity. Then the next application of $\mathbf{A}$, formally representing a strike by the left end, in fact calculates the results of a strike at the right end, with the velocities assigned to the 'wrong' end. $\mathbf{P}$ restores the velocities to the correct ends.
PA is applied once for each impact, and for odd impacts the entire product is premultiplied by $\mathbf{P}$ to reassign velocities to the correct ends (i.e., to cancel one excess $\mathbf{P}$, since $\mathbf{P}=\mathbf{P}^{-1}$ ).

The $i$ th impact refers to end $a$ if $i$ is odd, and presumes that $v_{a, i-1}<0$. It refers to end $b$ if $i$ is even, and in that case presumes that $v_{b, i-1}<0$. In general,

$$
\mathbf{v}_{i}= \begin{cases}\mathbf{P}(\mathbf{P A})^{i} \mathbf{v}_{0} \text { if } i \text { is odd } \\ (\mathbf{P A})^{i} \mathbf{v}_{0} & \text { if } i \text { is even }\end{cases}
$$

Equation (1) is the main equation from which all other clattering quantities (e.g. total number of impacts in the clattering sequence, velocity changes, impulses, time for clattering, forces, pulse durations, etc.) are derived. Observe that the only system parameters to occur in Eq. (1) are $q$ and $e_{e}$.

Our main goal in this paper is to describe the velocity changes during clattering for every point in the entire $\left[q, e_{e}\right]$ plane, that is, in global parameter space. Every possible bar configuration (in terms of mass distribution and coefficient of restitution) within the assumptions of our model corresponds to a point, or a $\left(q, e_{e}\right)$ pair, in the above global design space. For initial conditions we will generally take $\mathbf{v}_{0} \equiv\left[-v_{0},-v_{0}\right]$, where $v_{0}$ is a positive number. However, as shown in [7] the results of clattering with other initial conditions are easily derivable from the 'pure translation' initial condition. (Most of the calculations, and the resulting plots, presented in this paper were obtained with the software package Mathematica [16].)

\section{Parameters pertinent for assessing shock damage}

Damage can occur when the high forces due to impact exceed structural strengths of the product, or when internally suspended components are highly stressed 
by the sudden violent oscillations. The latter, called shock-response $([13,14,5,3])$, is central to electronic product durability because of the importance of suspended fragile components like circuit packages (ICs), displays, and disk drives, etc., to product functioning. (Note that a populated printed circuit board (PCB) can be considered as a suspended system due to its flexibility.)

Shock response is conventionally taken to be the force (or acceleration) experienced by an elastically suspended fragile mass, due to a shock-pulse experienced at its suspension point. It turns out that shock response depends heavily on the time-scales of the suspension-point shock pulse (rise-time, pulse duration, time intervals between pulses) as they relate to the suspended system's natural oscillation period; as well as either the shock pulse's magnitude (peak acceleration) or its area (that is, change in velocity, $\Delta v$ ). Since clattering involves transmutation of the overall impact into a much longer sequence of brief pulses, additional time scales have to be considered for shock analysis.

We use a simple rigid-body model in our analysis that ignores structural and material details of the product. Hence we shall confine ourselves to the consideration of velocity shocks - high magnitude acceleration pulses considerably shorter in duration that the suspended system's oscillation period, whose net effect is to impart an 'instantaneous velocity change' to the base of the suspended fragile sub-system. (A velocity shock can always be converted into a more detailed acceleration-pulse by modeling material and structual deformation.) However, by considering objects of finite extent (as opposed to point masses), we show that 'proper location' of the fragile component is important in increasing product durability, along with the traditional techniques of controlling natural frequencies of suspended components, eliminating relative motion or restricting it to prevent component slap.

There are two aspects of a sequence of velocity shocks that are relevant here: (1) the time duration between the individual shocks, and, (2) the magnitude of the largest impact. Figure 2, reproduced from [7], shows the total number of impacts for any value of $\left(q, e_{e}\right)$. The time durations between these impacts are plotted in detail in [7]. If the time separation between the shocks is considerably longer than the response time of the suspended component (so that suspension vibrations have a chance to die away), then the largest individual impact is important; if they are comparable then damaging resonances can occur; and if the entire clattering sequence is completed in less than one suspension period, then the details of the sequence do not matter and only the net change in velocity (sum of all the individual velocity shocks) counts. (Ideally, to assess the damage potential of repeated shocks, one should either 'drive' a model of the system with multiple shock pulses separated by the sequence of time durations obtained in [7], or determine it experimentally.)

We now calculate the largest velocity shocks at all locations on the bar.

\section{Maximum velocity changes along bar}

As a result of the eccentric impacts during clattering, the bar experiences two kinds of velocity changes, translational and rotational. For every impact, the rotational shock is the same for the entire bar. However, every point on the bar undergoes a different translational velocity change. The $\mathrm{CP}$ suffers no change in translational velocity during impact, and the change in velocity at other points varies linearly as their distance from the CP. Naturally the ends of the bar, being furthest away from the $\mathrm{CP}$, experience maximal velocity shocks. For a given impact, points between the impacting end and the $\mathrm{CP}$ experience positive velocity shocks and points on the other side of the $\mathrm{CP}$ experience negative, or downwards, velocity shocks. The velocity shock at the CM is the mean of that at the two ends.

The aim of safe component placement is to find a region of the bar where the greatest velocity shocks are less than anywhere else. Obviously for an impact at end $a$, components at the corresponding center of percussion, $P_{a}$, experience no shock. But for an impact at end $b$, which is equally likely, components at $P_{a}$ will experience a fairly large shock whereas the components at the center of percussion, $P_{b}$, will see no shock. Therefore when all possible impacts are considered, the center of the bar is the safest choice.

To summarize, during clattering of a given bar, its ends experience the largest velocity shock and its middle the least maximal velocity shock. For points inbetween, the largest velocity shock is obtained through linear interpolation of the largest velocity shocks at the middle and the ends of the bar.

From Eq. (1), it can be shown that the velocity change at the ends due to the $i$ th impact is given as:

$$
\begin{aligned}
\Delta \mathbf{v}_{i}= & {\left[\begin{array}{c}
\Delta v_{a, i} \\
\Delta v_{b, i}
\end{array}\right] } \\
= & \left\{\begin{array}{c}
(\mathbf{A}-\mathbf{I})(\mathbf{P A})^{i-1} \mathbf{v}_{0} \\
\text { if } i \text { is odd } \\
\mathbf{P}(\mathbf{A}-\mathbf{I})(\mathbf{P A})^{i-1} \mathbf{v}_{0} \\
\text { if } i \text { is even }
\end{array}\right.
\end{aligned}
$$




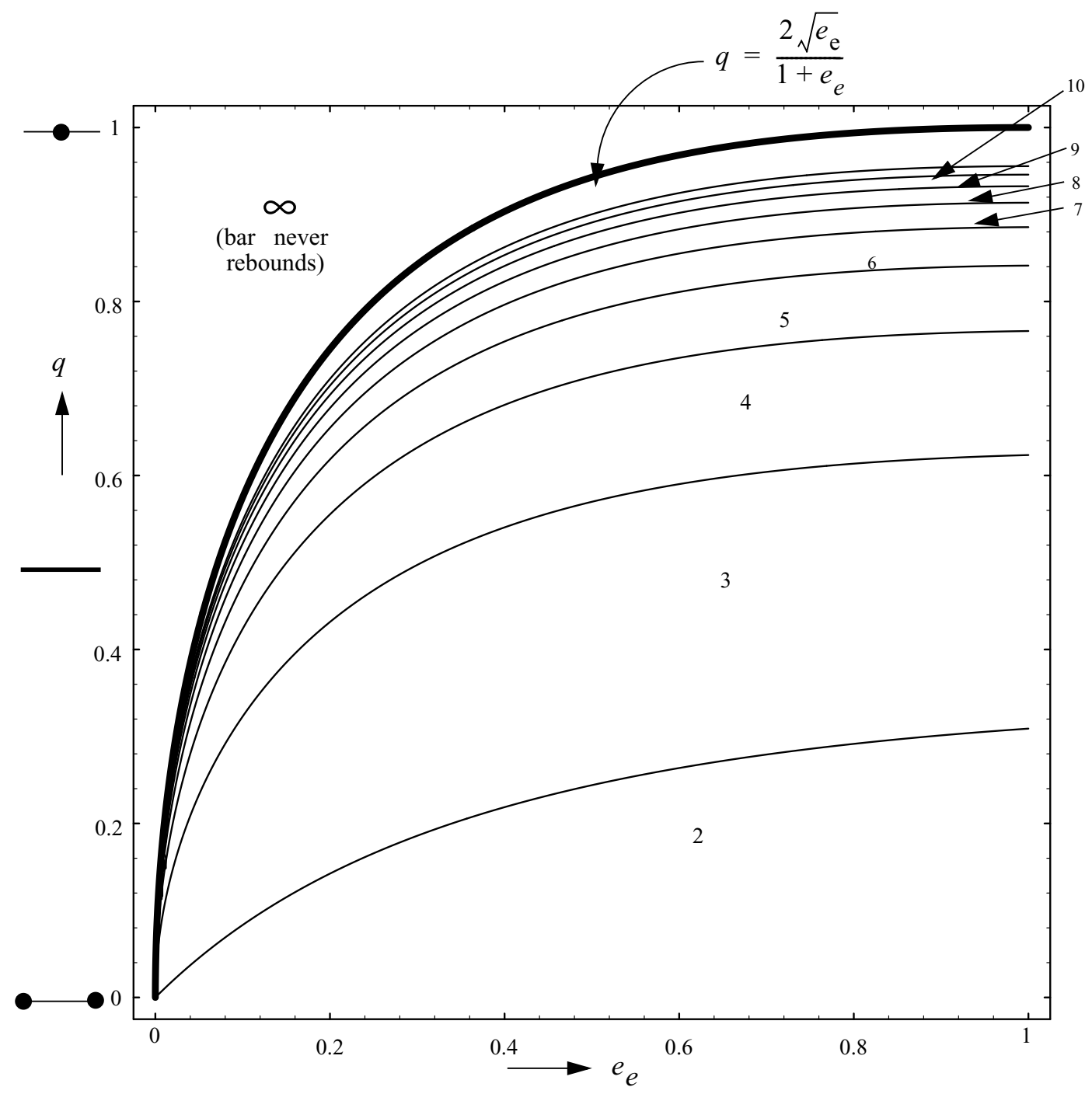

Fig. 2. Contour-plot of the number of impacts in the $\left[q, e_{e}\right]$ plane. (Mass distributions corresponding to $q=0,0.5$ and 1 are illustrated schematically on the left of the $q$ axis.) The fewest number of impacts occurs for high restitution and high moment of inertia - the minimum is two. The dominant region has 3 impacts, including the most common real-world mass distributions (i.e., small deviations from uniformly distributed mass). There is a significant area outside the bounding curve $q=2 \sqrt{e_{e}} /\left(1+e_{e}\right)$, where the bar experiences an infinite number of impacts in finite time and never rebounds.

where $\mathbf{I}$ is the $2 \times 2$ identity matrix. The changes in angular velocity, CM velocity, and the velocity at any location at a distance $l$ from the center of the bar (as shown in Fig. 1) may be found via operations on $\Delta \mathbf{v}_{i}$ :

$$
\begin{aligned}
\Delta v_{l, i} & =\left[\frac{L-l}{2 L}, \frac{L+l}{2 L}\right] * \Delta \mathbf{v}_{i}, \\
\Delta v_{c, i} & =\left[\frac{1}{2}, \frac{1}{2}\right] * \Delta \mathbf{v}_{i} \\
\Delta \omega_{i} & =\left[-\frac{1}{2 L}, \frac{1}{2 L}\right] * \Delta \mathbf{v}_{i}
\end{aligned}
$$

So, given specific values $\left(q, e_{e}\right)$ and initial velocity components, we can determine the entire sequence of velocity jumps at any point on the bar as it clatters.

The largest velocity shock at the ends of the bar, $\Delta v_{L}^{\max }$, can easily be found from the sequence obtained through Eq. (3); and then we can determine other quantities as:

$$
\begin{aligned}
\left|\Delta \omega^{\max }\right| & =(1+q) \frac{\Delta v_{L}^{\max }}{2 L}, \\
\Delta v_{c}^{\max } & =(1-q) \frac{\Delta v_{L}^{\max }}{2},
\end{aligned}
$$




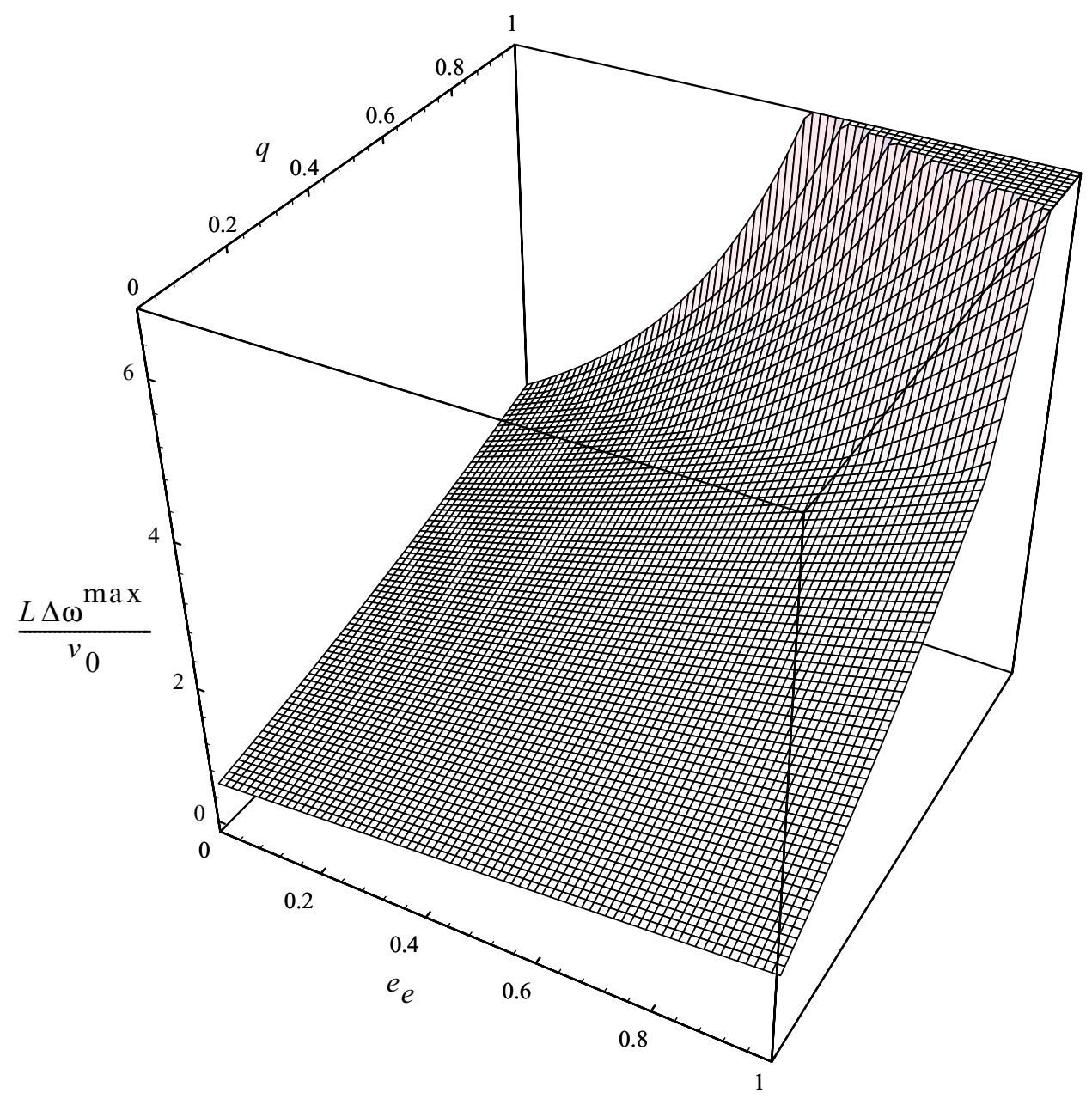

Fig. 3. 3-D surface-plot of the maximum angular velocity change in any one impact $\Delta \omega^{\max }$, normalized by $v_{0} / L$. Angular velocity and its changes are the same at all points on the bar.

$$
\Delta v_{l}^{\max }=\left[(1-q)+\frac{l}{L}(1+q)\right] \frac{\Delta v_{L}^{\max }}{2}
$$

Note that $\Delta v_{c}^{\max } \leqslant \frac{1}{2} \Delta v^{\max }$. Figure 3 shows the absolute values of the maximum change in angular velocities, $\left|\Delta \omega^{\max }\right|$.

In terms of a positional parameter $s \equiv l / L$, our aim is to calculate $\Delta v_{s}^{\max }$ for $0 \leqslant s \leqslant 1$ in order to know the maximal velocity shocks at all locations of the bar. (Note that a center-symmetric mass distribution has been assumed in our model, $s=0$ corresponds to the $\mathrm{CM}$ and $s=1$ represents the ends of the bar.) Figure 4 shows a 3-D surface-plot of the normalized maximum velocity changes, $\Delta v_{s}^{\max } / v_{0}$, for $s=0,0.5$, and 1 , in the entire $\left[q, e_{e}\right]$ design space. The plots for $s=0$ and $s=1$ represent the lower and the upper bound on the plots for any other location $0 \leqslant s \leqslant 1$ on the bar. The plot for $s=1$ is monotonic with increasing $q$ and $e_{e}$, whereas the plot for $s=0$ is not.

However, all three plots, for $s=0,0.5$, and 1 show that the velocity shocks decrease monotonically with decreasing $e_{e}$. This implies that $e_{e}$ should be minimized for reducing velocity shocks, that is, the products' hard casing - that gives rise to the short duration shock-pulses - should be designed in terms of materials and structure to absorb maximum energy during impact.

A key observation to be made from the plots of Fig. 4 is that the value of the normalized individual velocity shocks varies dramatically in the $\left[q, e_{e}\right]$ plane and is significantly higher, or lower, than 2.0 in several parts. This is in stark contrast to clatter-free impacts in standardized testing $([11,10,12,1])$ where a maximal value of $2 v_{0}$ is assumed for velocity shock along the entire 


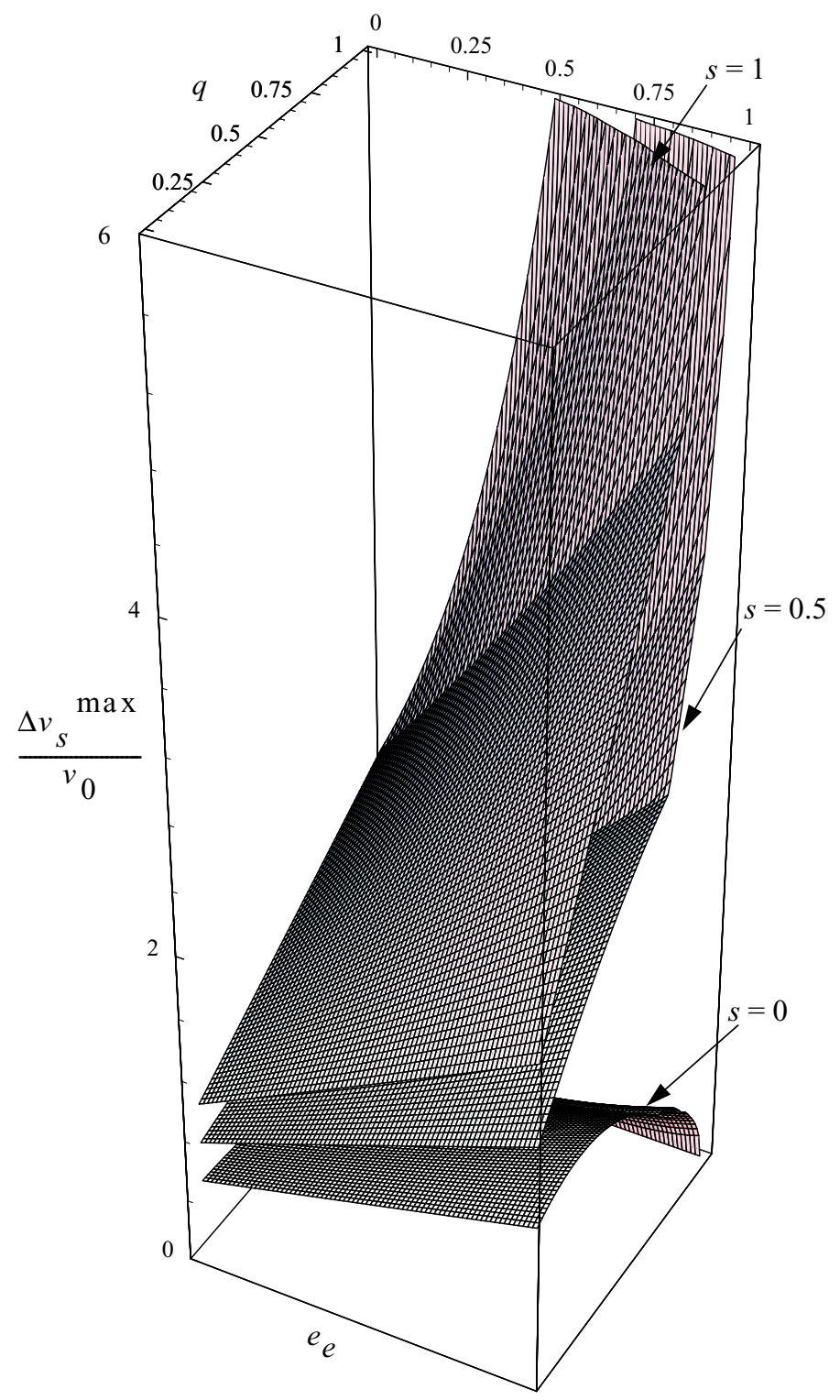

Fig. 4. Plot of normalized maximal velocity change $\Delta v_{s}^{\max } / v_{0}$ during clattering for the ends $(s=1)$, middle $(s=0)$, and one-quarter points $(s=0.5)$ of the bar, as a function of $\left(q, e_{e}\right)$. The greatest velocity shocks are experienced by the ends, and the least, in the middle of the bar. So $s=0$ and $s=1$ represent the lower and upper bound on the plots for $0 \leqslant s \leqslant 1$. Observe that the plot for $s=1$ is monotonic with increasing $q$ and $e_{e}$, while for $s=0$ it is not. The plots are fairly smooth except for regions in the vicinity of $q=1$ where they rise, or dip, sharply. The large gap between the $s=0$ and $s=1$ plots for higher values of $q$, coupled with the dip in the $s=0$ plot, implies that a zone of lower velocity shocks is created at the center of the bar at the expense of a zone of amplified velocity shocks at the ends of the bar. The plot for $s=0.5$ is presented as an example; similar plots can be obtained for any other location by linear combination of the plots for $s=0$ and $s=1$.

product ([5]). The above velocity amplifications, and reductions, at various locations along the bar during clattering are the major findings of our study. In particular, for higher values of $q$, it can be seen that the center of the bar, and its vicinity, experience significantly lower velocity shocks whereas the ends experience fairly high velocity shocks. In this regard, clatter- ing acts to 'cushion' the center of the bar. This fact has useful design implications.

In principle, using Eq. (5), plots like those shown in Fig. 4 can be obtained for any location on the bar and the maximal velocity shock at that location determined as a function of $\left(q, e_{e}\right)$. However, for purposes of product design it is easier, and more useful, to view the $3 \mathrm{D}$ 


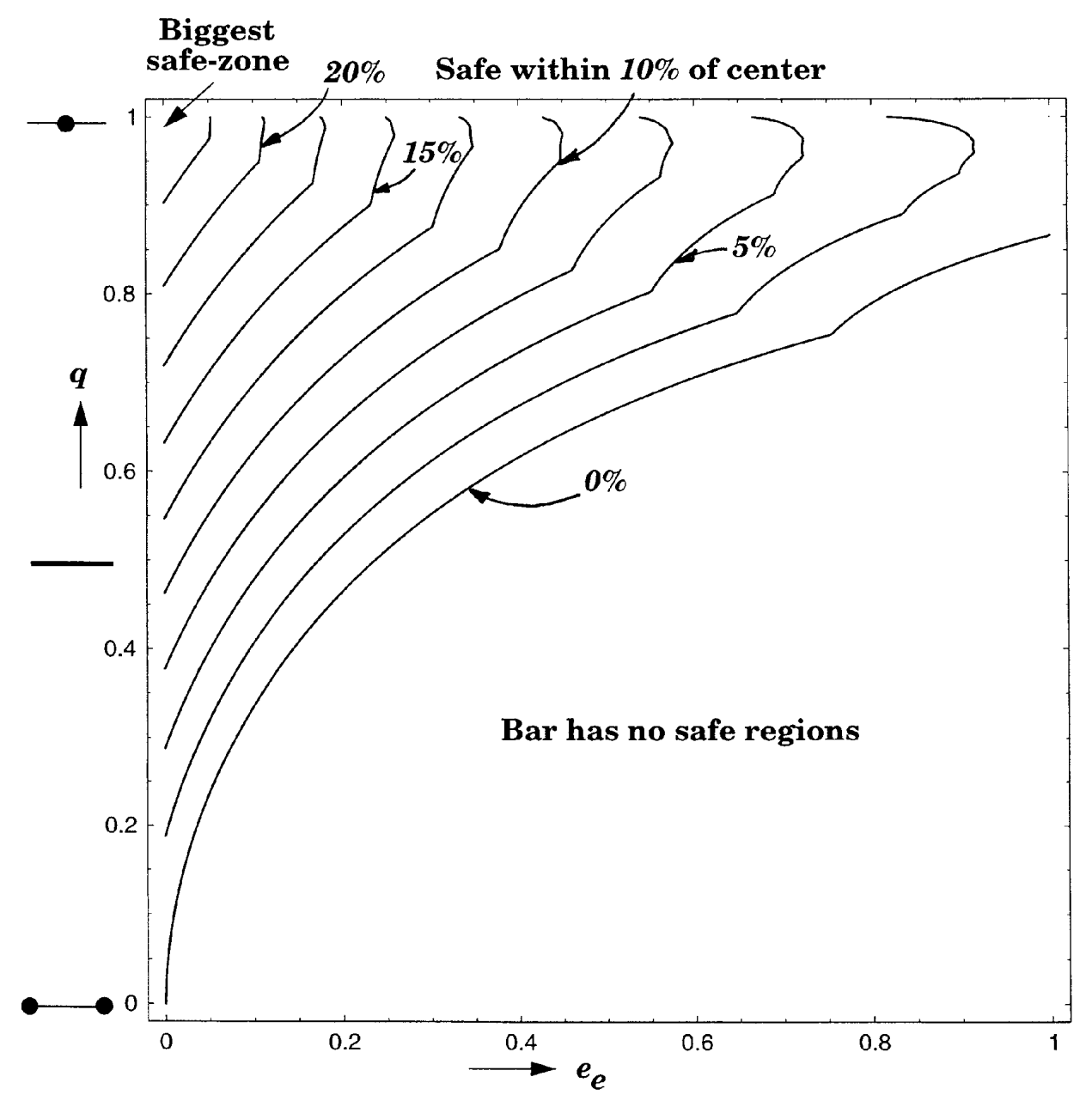

Fig. 5. Contour-plots of constant values of $s$ for velocity shock thresholds, $\Delta v_{s}^{\max }=v_{0} / 2$ during clattering. The values of the parameter $s$ are presented in terms of percentage of the bar from its center. The plot shows that only half of the $\left[q, e_{e}\right]$ design space provides bar configurations to meet the above threshold. For a given $e_{e}$, the extent of the safe-zone can be increased by increasing $q$.

surface-plots of Fig. 4 in the form of contour-plots of $s$ in the $\left[q, e_{e}\right]$ plane for constant values of $\Delta v_{s}^{\max } / v_{0}$.

\section{Design methology and guidelines}

The goal of the product designer is to figure out an optimal (and achievable) value of $\left(q, e_{e}\right)$ so that the corresponding object provides safe regions for the placement of fragile components where the components' fragility - in terms of tolerable level of velocity shock will not be exceeded during clattering. To calculate regions of the bar, in the $\left[q, e_{e}\right]$ plane, where given velocity shock thresholds are not exceeded, Eq. (5) can be recast to yield:

$$
s=\frac{1}{1+q}\left[\frac{2 \Delta v_{s}^{\max }}{\Delta v_{L}^{\max }}+q-1\right]
$$

Depending on the desired maximal velocity shock threshold, Eq. 5 can be plotted to yield contour-plots of $s$ in the $\left[q, e_{e}\right]$ plane and the plots examined to determine if there exists a safe enough region that would accomodate the fragile component.

For example, let's say that the design goal is to find a bar configuration with regions that experience, at most, a maximal velocity shock of only half the impact velocity $v_{0}$, and, substantially most of the bar does not experience velocity shocks exceeding twice the impact velocity. The second part of the design goal is necessary because while a high value of $q$ can help protect components near the center of mass, it may hurt those near the ends (both because velocity jumps are 


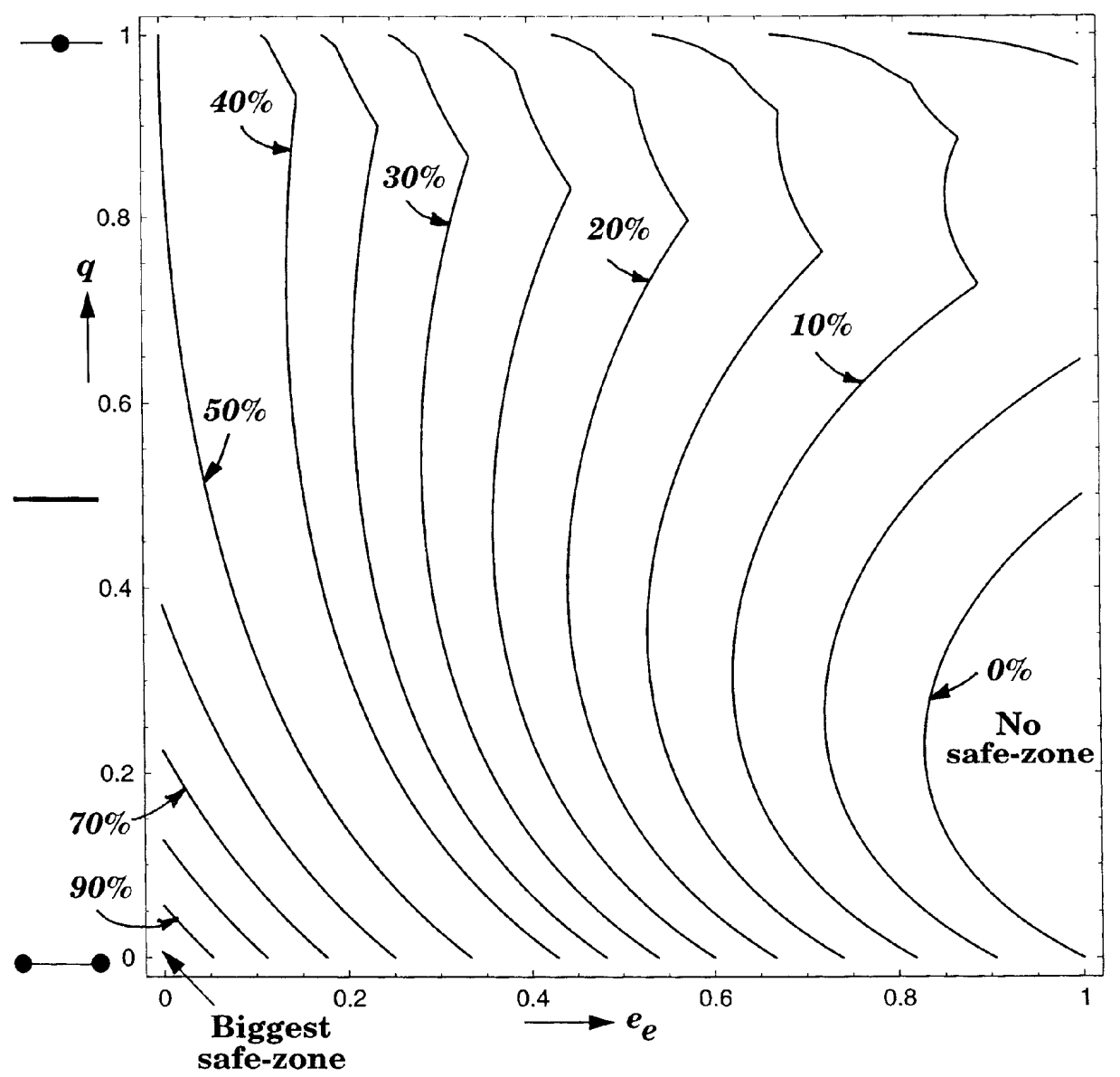

Fig. 6. Contour-plots of constant values of $s$ for maximal velocity shock thresholds, $\Delta v_{s}^{\max }=v_{0}$ during clattering.

large, and because the additional high-frequency reversals could cause resonance). Figures 5, 6 and 7 show contour-plots of constant values of $s$ for maximal velocity shock thresholds, $\Delta v_{s}^{\max }=v_{0} / 2, v_{0}$ and $2 v_{0}$ respectively. The values of the parameter $s$ are presented in terms of percentage of the bar from its center. (That is, $s=l / L=0.1$ represents a region around the center of the bar that is equal to $10 \%$ of its total length, $s=0.2=20 \%$ of the bar, and so on.)

Once again, as with Figs 3 and 4, Figs 5, 6 and 7 illustrate that for a given value of $q$, the extent of the safe-zone can be increased by decreasing $e_{e}$. However, the figures differ both quantitatively and qualitatively in terms of the effect of $q$.

Observe in Fig. 5 that roughly only half of the global design space provides bar configurations that have regions experiencing $\Delta v_{s}^{\max }=v_{0} / 2$. For $\left(q, e_{e}\right)$ that provide $v_{0} / 2$ shock-threshold zones, increasing $q$ only increases the extent of the safe-zone.
Figure 6 shows that most of design space, except for a small region at bottom right, provides bar configurations that have safe-zones experiencing maximal velocity shocks $\Delta v_{s}^{\max }=v_{0}$ during clattering. Note, however, that the behaviour with respect to $q$ is not monotonic like in Fig. 5. For instance, in some parts of the $\left[q, e_{e}\right]$ plane, the extent of a safe-zone can be increased by both increasing and decreasing $q$, and in other parts changing $q$ has no appreciable effect on the size of the safe-zone.

Figure 7 shows that the entire design space provides regions on the bar that experience velocity shocks of at most $2 v_{0}$. The behaviour with respect to $q$ is monotonic. However, unlike Fig. 5, for a given $e_{e}$, the extent of the safe-zone increases by decreasing $q$. As mentioned earlier, this is because for higher values of $q$ the ends of the bar experience large velocity changes, and velocity reversals, during clattering.

In terms of available design choices for $\left(q, e_{e}\right)$, there are additional limitations. $q$ can be increased for a 


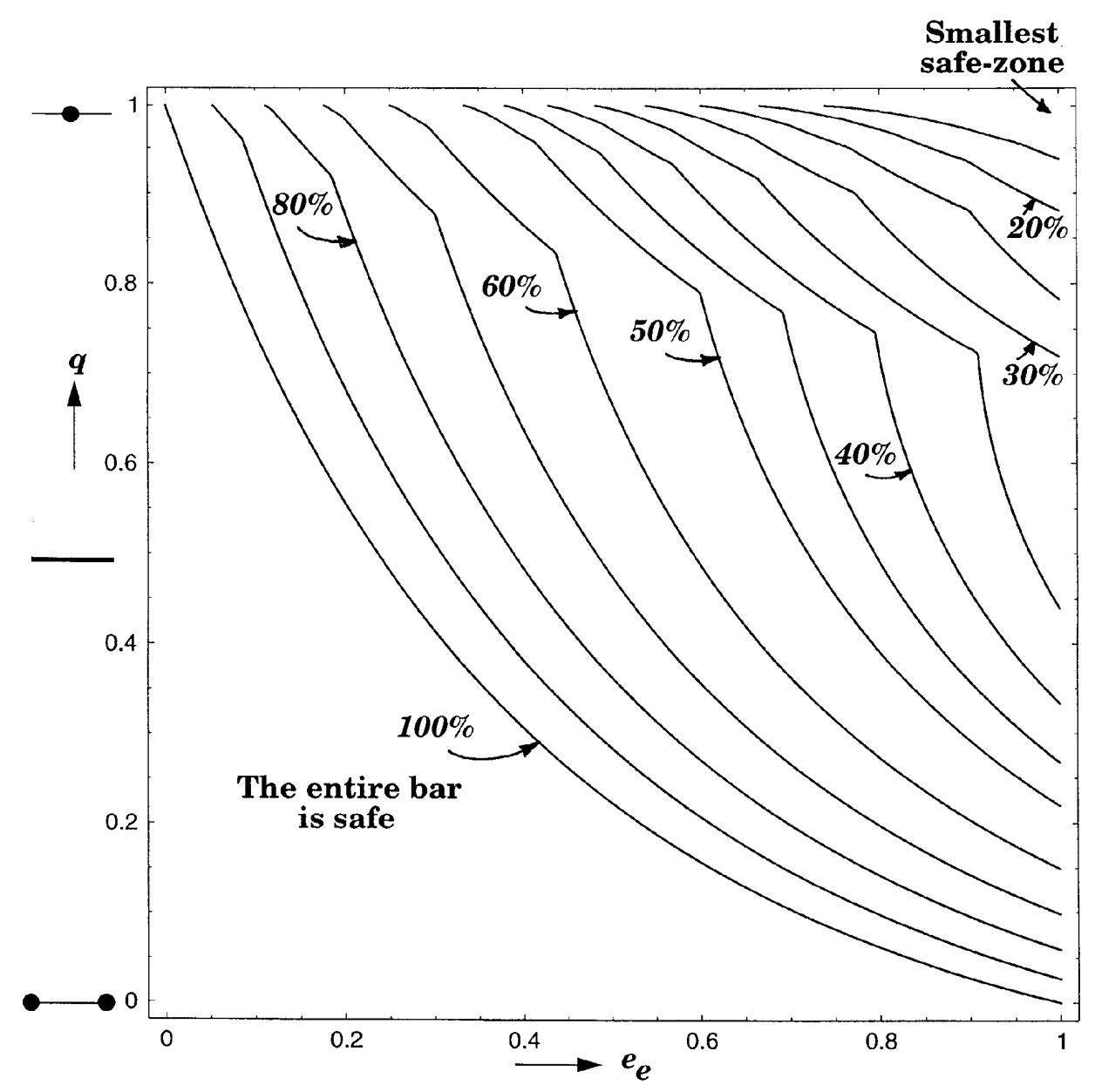

Fig. 7. Contour-plots of constant values of $s$ for maximal velocity shock thresholds, $\Delta v_{s}^{\max }=2 v_{0}$ during clattering. The plot shows that the entire design provides bar configurations to meet the above threshold. For a given $\varepsilon_{e}$, the extent of the safe-zone can be increased by decreasing $q$.

product by placing heavier components (for example, the battery in a cellular phone), and even superfluos mass, towards the middle. However, for size-optimized portable electronic products with their dense packaging of components and optimal usage of all available internal space, a large $q$ is practically infeasible. Similarly, it is rather difficult to make products that would be very bouncy (high $e_{e}$ ) or 'very dead' (very low $e_{e}$ ) during impact ([8]). The final choice has to be product-specific. In view of the above, four pairs of $\left(q, e_{e}\right)$ are examined in Table 1. The first one, $\left(q, e_{e}\right)=(0.5,0.3)$, is the canonical reference configuration with uniform mass distribution and moderate damping; and the other three, $\left(q, e_{e}\right)=(0.73,0.2),(0.67,0.15)$ and $(0.57,0.1)$ are suggested configurations. Between them the three suggested configurations illustrate, independently, the effects of lowering $e_{e}$ and increasing $q$.
Table 1 lists values of the extent of the safe-zones for arbitrary velocity-shock thresholds of $v_{0} / 2, v_{0}$ and $2 v_{0}$, maximum velocity shock at the end of the bar, maximum angular velocity change, total velocity change at the center of the bar, and the total number of impacts during clattering, for each of the above four $\left(q, e_{e}\right)$ pairs.

It can be seen from Table 1 that the reference configuration does not have the reqired safe-zone in the middle of the bar. Both $\left(q, e_{e}\right)=(0.73,0.2)$ and $(0.67,0.15)$ provide a $10 \%$ region on the bar where the maximal velocity shock does not exceed $v_{0} / 2$, with small regions towards the ends of the bar that experience shocks slightly greater than $2 v_{0} . \quad\left(q, e_{e}\right)=(0.57,0.1)$ provides a somewhat smaller safe-zone but has no region that experiences greater than $2 v_{0}$. (If the fragile components being protected are sensitive to rotational shock, 
Table 1

Some example product configurations in terms of values of $\left(q, e_{e}\right)$ that yield, roughly, a $10 \%$ safe zone in the middle of the bar where the velocity shock is less that $v_{0} / 2$ while substantially the rest of the bar does not experience shocks exceeding $2 v_{0}$. The first configuration, $\left(q, e_{e}\right)=(0.5,0.3)$, is a reference configuration (with unifrom mass distribution) that does not have the required safe zone

\begin{tabular}{lcccc}
\hline$\left(q, e_{e}\right)=$ & $(0.5,0.3)$ & $(0.73,0.2)$ & $(0.67,0.15)$ & $(0.57,0.1)$ \\
\hline$s\left(\Delta v^{\max } / v_{0}=0.5\right)$ & $0 \%$ & $10.1 \%$ & $9.6 \%$ & $8.2 \%$ \\
$s\left(\Delta v^{\max } / v_{0}=1.0\right)$ & $28.8 \%$ & $35.7 \%$ & $39.1 \%$ & $43.8 \%$ \\
$s\left(\Delta v^{\max } / v_{0}=2.0\right)$ & $90.9 \%$ & $87.1 \%$ & $97.9 \%$ & $100 \%$ \\
$\Delta v_{L}^{\max } / v_{0}$ & 2.145 & 2.251 & 2.036 & 1.790 \\
$L \Delta \omega^{\max } / v_{0}$ & 1.609 & 1.947 & 1.7 & 1.405 \\
$\Delta v_{c}^{\text {total }} / v_{0}$ & 1.115 & 1.0 & 1.0 & 1.0 \\
No. of impacts & 4 & 16 & 31 & 24 \\
\hline
\end{tabular}

then the rotational stiffness of their suspensions has to be evaluated in comparison to the angular velocity changes.)

\section{Summation}

Clattering motion results when flat objects strike the ground at an angle, subjecting the object to a rapid sequence of impacts and velocity reversals. The magnitude, periodicity, and duration of impact loads play a major role in their ability to cause shock damage on suspended fragile components. We have analyzed clattering motion using the equations of motion derived in [6,7], and obtained the maximum velocity changes occuring along all locations of a unidimensional bar clattering in two dimensions. (The magnitude of the change in velocity determines the impact forces experienced by 'softly suspended' components.)

We have shown that as a result of clattering, parts of the product can experience velocity changes that are either substantially higher or lower than those encountered in a single clatter-free impact. We examined this fact in terms of its design implications for improving the drop-tolerance of portable products, via the creation of regions on the product - through adjustment of its mass distribution and coefficient of restitution that experience velocity shocks that are far lower than the impact velocity. We showed that, in general, product ruggedness can be increased by designing its hard casing to maximize energy dissipation during impact and by configuring the product to have low moment of inertia.

We also showed, as an example, how to choose product configurations that would yield a $10 \%$ region in the middle, to mount fragile components, where the velocity shocks would not exceed half the impact velocity, while making sure that substantially most of the rest of the product would not see shocks exceeding twice the impact velocity.

\section{References}

[1] ASTM D3332-88, Standard Test Methods for MechanicalShock Fragility of Products, Using Shock Machines, Annual Book of ASTM Standards, 15.09, American Society of Testing and Materials, Philadelphia, 1993.

[2] W. Goldsmith, The Theory and Physical Behaviour of Colliding Solids, Edward Arnold Publishers Ltd., London, 1960.

[3] S. Goyal, E. Buratynski and G. Elko, Role of Shock Response Spectrum in Electronic Product Suspension Design, International J. of Microcircuits \& Electronic Packaging 23(2) (2000), 182-190.

[4] S. Goyal and E. Buratynski, Methods for Realistic DropTesting, International J. of Microcircuits \& Electronic Packaging 23(1) (2000), 45-52.

[5] S. Goyal, J.M. Papadopoulos and P.A. Sullivan, Shock Protection of Portable Electronic Products: Shock Response Spectrum, Damage Boundary Approach, and Beyond, Shock and Vibration, 4(3) (1997), 169-191.

[6] S. Goyal, J.M. Papadopoulos and P.A. Sullivan, The Dynamics of Clattering I: Equation of Motion and Examples, Journal of Dynamic Systems, Measurement, and Control 120(1) (1998), 83-93.

[7] S. Goyal, J.M. Papadopoulos and P.A. Sullivan, The Dynamics of Clattering II: Global Results and Shock Protection', Journal of Dynamic Systems, Measurement, and Control 120(1) (1998), 94-102.

[8] S. Goyal, R.G. Larson and C.J. Aloisio, Quantitative Prediction of Impact Forces in Elastomers, Journal of Engineering Materials and Technology 121(3) (1999), 294-304.

[9] S. Goyal, S. Upasani and D.M. Patel, Improving Impact Tolerance of Portable Electronic Products: Case Study of Cellular Phones, Experimental Mechanics 39(1) (1999), 159-174.

[10] IEC 68-2-27, International Standard: Basic Enviromental Testing Procedures, International Electrotechnical Commission, Geneva, Switzerland, 1987.

[11] MIL-STD-883C, Military Standard, Test Methods and Procedures for Microelectronics, US Office of Naval Publications, Washington, DC, 1983.

[12] MIL-STD-810E, Military Standard, Environmental Test Methods and Engineering Guidelines, US Office of Naval Publications, Washington, DC, 1989. 
[13] R.D. Mindlin, Dynamics of Package Cushioning, Bell Systems Journal 24 (1945), 353-461.

[14] R.E. Newton, Theory of Shock Isolation', in Shock \& Vibration Handbook, McGraw-Hill Book Company, New York, 1988.

[15] D. Stoianovici and Y. Hurmuzlu, A Critical Study of the
Applicability of Rigid-Body Collision Theory, Journal of Applied Mechanics 63(2) (1996), 307-316.

[16] S. Wolfram, Mathematica: A System for Doing Mathematics by Computer, Addison-Wesley Publishing Company, Inc., New York, 1991 

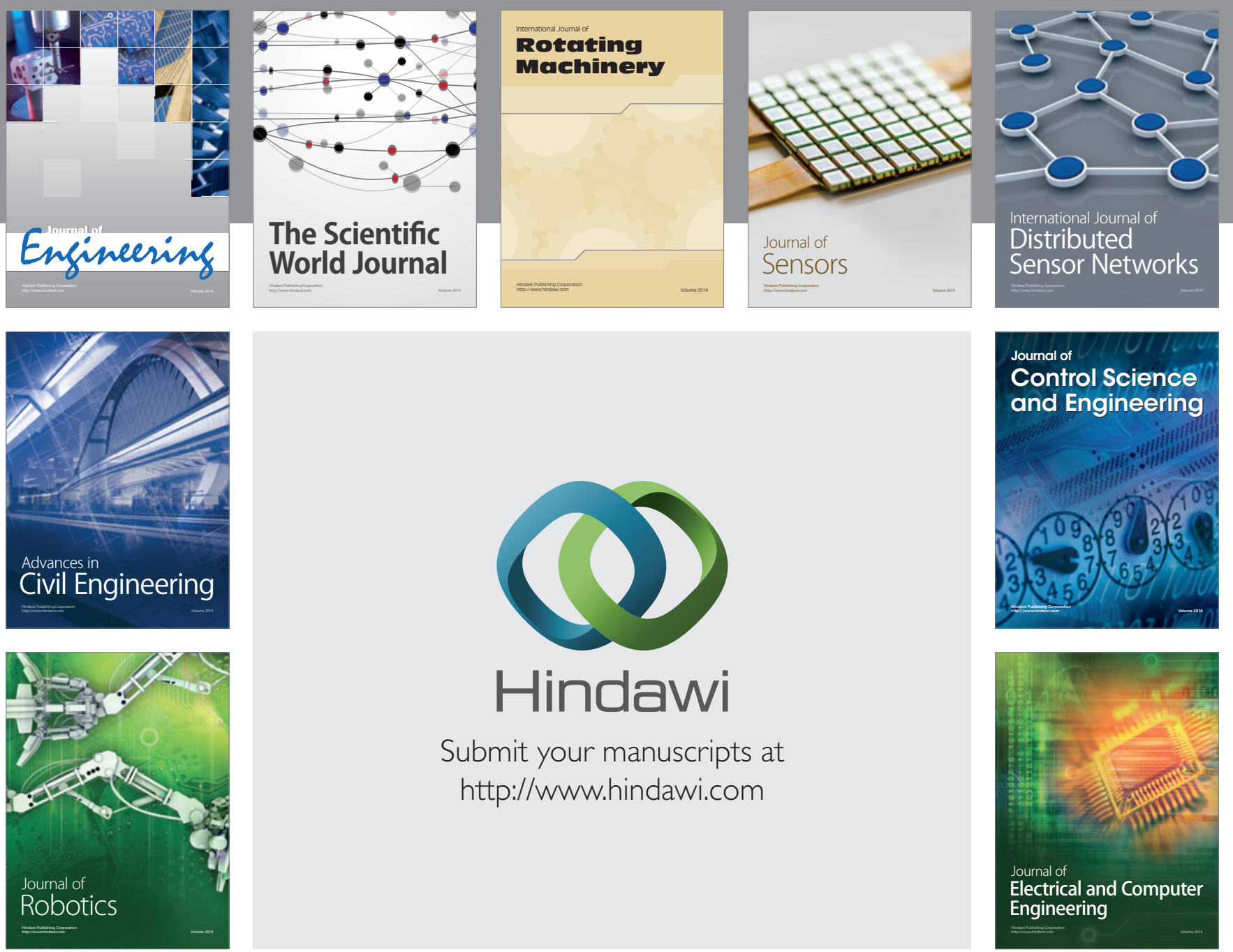

Submit your manuscripts at

http://www.hindawi.com
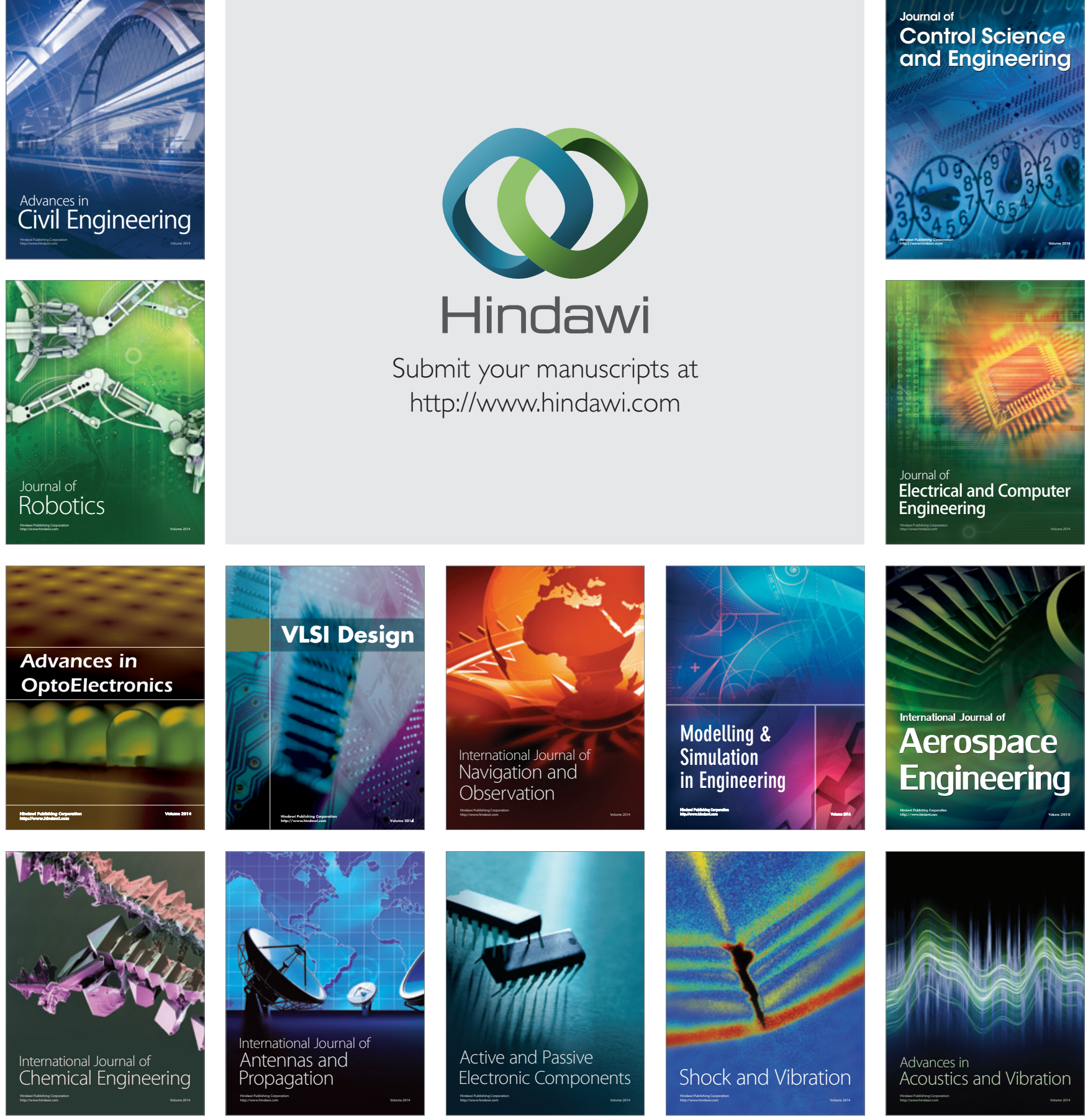